\title{
STAR FORMATION HISTORIES IN DWARF IRREGULAR GALAXIES IN THE LOCAL GROUP
}

\author{
L. GREGGIO ${ }^{1}$, G. MARCONI ${ }^{1}$, P. FOCARDI ${ }^{1}$, M. TOSI ${ }^{2}$ \\ 1 Astronomy Department, University of Bologna, CP 596, I-40100 Bologna, Italy \\ 2 Astronomical Observatory of Bologna, Via Zamboni 33, I-40100 Bologna, Italy
}

The process of star formation (SF) and the modalities with which it occurs in galaxies of different sizes are still poorly understood. On the other hand, interpretation of the chemical and photometric properties of galaxies requires the adoption of adequate laws for the SF rate and Initial Mass Function (IMF) in model computations. Dwarf irregular galaxies in the Local Group offer the chance to study their SF history through analysis of their HR diagrams, which can be derived down to $V \simeq 25$, corresponding to $M_{V \simeq 0}$, i.e. to main sequence stars of $\simeq 2 M_{\odot}$. Therefore, for these galaxies, we can derive information on the star formation activity which has occurred over the last 1 Gyr approximately. In this framework, we have undertaken CCD observations of Dwarf irregular galaxies in the Local Group with ESO telescopes and briefly present here the results obtained for DDO 210, Sextans B and NGC 3109.

For each galaxy, one or more regions were observed in the Johnson $\mathrm{B}, \mathrm{V}$ and $\mathrm{R}$ bands at the 2.2-m ESO-MPI telescope in La Silla (Chile) and the CCD data have been reduced with the DAOPHOT package (Stetson 1987). Interpretation of the derived Colour Magnitude Diagrams (CMD) was done by comparing corresponding Monte Carlo simulations of theoretical HR diagrams. In this procedure, only stars with photometric error smaller than 0.1 mag are taken into account. The simulations done for these galaxies are based on the evolutionary tracks by Bertilli $e t$ al. (1986), with $Z=0.001$, by Greggio (1984), and on empirical BC and colour calibrations, as functions of effective temperature, for main sequence, giant and supergiant stars (Johnson 1966, Flower 1977, Humphreys \& McElroy 1984, Elias et al. 1985). A random scatter for the photometric error and a completeness correction consistent with the corresponding observational values have been introduced in the simulations. This method (Tosi et al. in prep.) is particularly effective in accounting for the stochastic nature of the SF process, the effects of the small number statistics and the spread introduced by photometric errors.

The brightest members of DDO 210 have magnitudes in the range $21.5 \leq \mathrm{V} \leq 20.5$; the uncertainty is due to conspicuous contamination from both foreground stars and background galaxies. Adopting a distance modulus of $25 \mathrm{mag}$, the brightest blue stars in this galaxy have $\mathrm{M}_{V \simeq-4 \text {, }}$ corresponding to either main sequence stars of $\simeq 8 \mathrm{M}_{e}$, or to core helium-burning objects of $\simeq 4 \mathrm{M}_{\Theta}$. The choice between the two is made difficult by the relatively red colour $(B-V \simeq 0.1)$ of these stars, but if they are blue supergiants we expect the clump of the corresponding main sequence stars to appear in CCD frames 1-2 mag deeper. Indeed, we have just taken deeper CCD frames of this galaxy and the data reductions are under way.

The CMD derived for Sextans B is shown in Fig. 1a, while Fig. 1b shows one of the simulated diagrams which is in better agreement with the observational data, having adopted a distance modulus of $26.1 \mathrm{mag}$. Fig. 1b was derived using a Salpeter IMF, with an upper mass cut-off of 

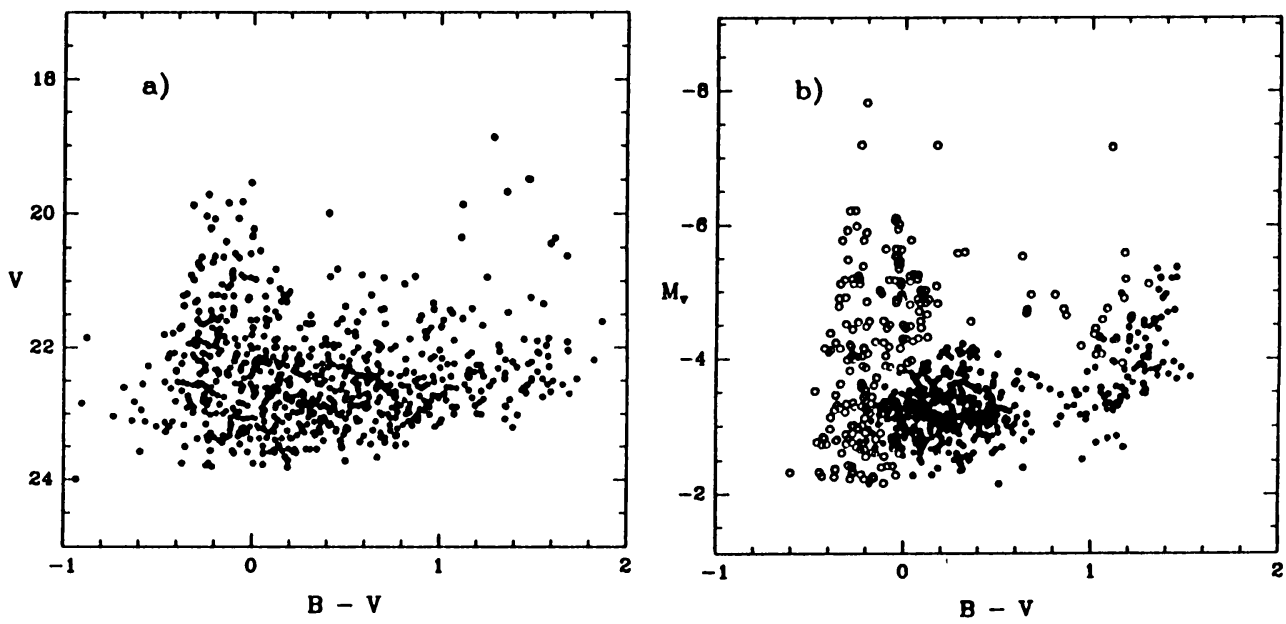

Figure 1. CMD for Sextans B (Fig. 1a) and a corresponding simulation (Fig. 1b) consisting of two bursts of SF. Only objects with photometric error smaller than 0.1 mag have been retained.

$120 \mathrm{M}_{\mathrm{e}}$, and two bursts of star formation: the first started $1 \mathrm{Gyr}$ ago and lasted $8.5 \times 10^{8} \mathrm{yr}$ (dots); the second started $1.2 \times 10^{8}$ yr ago and stopped $2 \times 10^{6}$ yr ago (circles). This particular choice was forced by the characteristics of the observational diagrams: a constant SF rate over the last $1 \mathrm{Gyr}$, or a recent burst continued up to now, would imply the presence of too many bright blue stars in the diagram, which are not seen in Fig. 1a.

NGC 3109 was only recently observed and reduced and we present very preliminary results for this galaxy. Its CMD shows the presence of very bright blue stars, extending up to $\mathbf{M}_{V \simeq-8}$, for an adopted distance modulus of $26.4 \mathrm{mag}$. This indicates a recent SF event. However, a constant SF rate over the last $1 \mathrm{Gyr}$ yields a synthetic HR diagram in which the main sequence stars are well separated from the blue supergiants, for $M_{V} \leq-6$, unlike the observational evidence. A number of simulations, varying both the SF rate and IMF, have been performed, but no satisfactory result has been obtained. Perhaps the assumption of a higher metal content would lead to a better agreement.

In all of the cases examined we found indications of recent, but not continuing star formation, seeming to support the idea that Dwarf galaxies experience bursting, rather than continuous SF. Closer scrutiny is needed to reach firm conclusions. In particular, comparing theoretical with observed luminosity functions will provide an independent check on the IMF. Further theoretical work and data acquisition are in progress.

\section{References}

Bertelli, G., Bressan, A., Chiosi, C., Angerer, K. (1986), Astron. Astrophys. Suppl. Ser. 66, 191. Elias, J.H., Frogel, J.A., Humphreys, R.M. (1985), Astrophys. J. Suppl. Ser. 57, 91.

Flower, P.J. (1977), Astron. Astrophys. 54, 31.

Greggio, L. (1984), Stellar Nucleosynthesis, C. Chiosi, A. Renzini (eds.) (Reidel: Dordrecht) p.137.

Humphreys, R.M., McElroy, D.B. (1984), Astrophys. J. 284, 565.

Johnson, H. (1966), Ann. Rev. Astron. Astrophys. 4, 193.

Stetson, P.B. (1987), Publ. Astron. Soc. Pac. 99, 191.

Tosi, M., Focardi, P., Greggio, L., Marconi, G., in preparation. 